\title{
Scrutiny on the produced spin current in spin circuit considering Nano- magnetic nodes and copper Nano-channel ${ }^{\#}$
}

\author{
S. N. Hosseinimotlagh*1, S.Ghaderi, H.Ghavidelfard
}

\section{Accepted $15^{\text {th }}$ August 2014}

Abstract: Electronics of spin or spintronics is a newfangled field which its purpose is to study the role of electron's spin in solid-state devices. spintronic devices require spin current. Spin current is a difference between spin-up and spin-down electric currents. This article reviews the spin current. In this paper, we intend to calculate spin current in two spin circuits' branches. Also we investigate simultaneously effects of nano-channel length and cross section area variations on it. Our findings show that spin current in series branch increase by simultaneously length of nano-channel reduction and rising of cross section area. For spin flip branches, we can reduce dissipation by simultaneously length and cross section area nano-channel reduction. We choose copper metal as nano-channel because of longer spin diffusion length, less spin current dissipation and equality of its lattice constant with those of permalloy.

Keywords: Circuit-spin-current-conduction-nano-channel.

\section{Introduction}

Spintronics studies the role of electron spin in solid-state devices. Spintronic devices require spin current [1]. Difference between spin-up and spin-down electric currents defines spin current. Comfortable way to create spin current is passing electron current form ferromagnetic materials. Spin injectors are materials which create spin-polarized electron injection. The most obvious spin injectors are ferromagnetic materials due to their high Curie temperatures, low coercivities and fast switching times. In this paper, we intend to calculate spin current in two spin circuits' branches. After an overview, for achieving this aim, we start with description of laws governing on spin circuits (section 2). Sections 3 and 6 cover the calculation of spin current in $\Pi$ and $T$ shaped spin circuit, respectively. Also in in section 6 the investigation simultaneously on the effects of nano-channel length and cross section area variations on spin current are addressed. Finally, we present conclusion and discussion in section 5

\section{Spin circuits}

Spin circuit is one of the circuits group in which flows spin current in addition to electron current. Such circuit exploits magnetic nodes as spin-polarized carries injectors and nonmagnetic channel as spin transporter between these magnetic nodes. From point of view Physics, magnetic node is defined as a collection of physical points in a device or a circuit where all the quantities of interest for spin and charge transport are at equilibrium [2]. Non-magnetic channel connects two magnetic nodes. Spin circuit obeys spin ohm's law and spin circuit theory. In compared with usual ohm's law, Spin ohm's law shows linear

${ }^{I}$ Department of Physics, Colleges of Sciences, Islamic Azad University, Shiraz, Iran

*Email: hoseinimotlagh@ hotmail.com

\# This paper has been presented at the International Conference on Advanced Technology\&Sciences (ICAT'14) held in Antalya (Turkey), August 12-15, 2014.

This journal is (c) Advanced Technology \& Science relation between spin current and spin voltage difference vector. Mathematical form of this law is [2]

$\overrightarrow{\mathrm{I}}_{\mathrm{s}}=\mathrm{G} \Delta \overrightarrow{\mathrm{V}}_{\mathrm{s}}$

Where $\overrightarrow{\mathrm{I}}_{\mathrm{S}}, \mathrm{G}, \Delta \overrightarrow{\mathrm{V}}_{\mathrm{s}}$ are spin current vector, spin conduction Matrix and spin voltage vector, respectively. Spin current and spin voltage vector are $3 * 1$ matrixes. The latter exists due tocreation of spin polarized population in the nano- magnetic node. Spin conduction matrix is defined as $3 * 3$ matrix proportionality constant which relates spin current vector to spin voltage difference vector and it can be written as [2]:

$G=\left[\begin{array}{lll}G_{11} & G_{12} & G_{13} \\ G_{21} & G_{22} & G_{23} \\ G_{31} & G_{32} & G_{33}\end{array}\right]$

Spin current vector in a branch of a spin circuit can be represented as

$\vec{I}_{\mathrm{s}}=\left[\begin{array}{c}\mathrm{I}_{\mathrm{sx}} \\ \mathrm{I}_{\mathrm{sy}} \\ \mathrm{I}_{\mathrm{sz}}\end{array}\right]$

Where $\mathrm{I}_{\mathrm{sx}}, \mathrm{I}_{\mathrm{sy}}$ and $\mathrm{I}_{\mathrm{sz}}$ is vector spin current components along $\mathrm{x}$ direction, $y$ - direction and $z-$ direction, respectively. Mathematical form spin voltage vector describe as

$\vec{V}_{\mathrm{s}}=\left[\begin{array}{c}\mathrm{V}_{\mathrm{sx}} \\ \mathrm{V}_{\mathrm{sy}} \\ \mathrm{V}_{\mathrm{sz}}\end{array}\right]$

Where $V_{s x}, V_{s y}$ and $V_{s z}$ is spin voltage vector components along $\mathrm{x}$ direction, y- direction and z- direction, respectively. Since spin currents are non-conservative, we must consider spin dissipation current to the virtual ground [3].

\section{II and T-shaped spin circuit}


In this section, we intend to calculate spin current in П-shaped spin circuit. As shown in Fig.1, our spin circuit consisting of two nano-magnetic nodes, $\mathrm{N}_{1}$ and $\mathrm{N}_{2}$, connected by a non-magnetic channel with cross section area A, resistivity $\rho$, length L, spin-flip length of the channel material $\lambda$. This circuit has two spin-flip branches and one series branch. Spin-flip branches model dissipation of spin current from the channel due to spin flip process which goes to zero spin voltage. We assume that this spin circuit has symmetric geometry and spin

Voltage vector's components are equal.

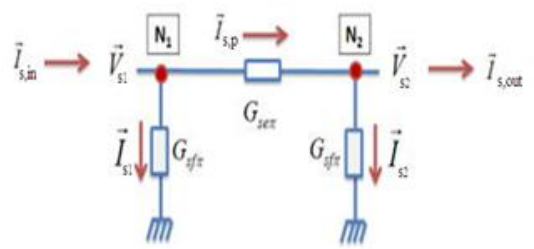

Fig. 1 П-shaped spin circuit with two nano-magnetic nodes and a nonmagnetic channel.

According to Kirchhoff's current law, we have [2]:

$\overrightarrow{\mathrm{I}}_{\mathrm{s}, \text { in }}=\overrightarrow{\mathrm{I}}_{\mathrm{sp}}-\overrightarrow{\mathrm{I}}_{\mathrm{s} 1}$

$\overrightarrow{\mathrm{I}}_{\mathrm{s}, \text { out }}=\overrightarrow{\mathrm{I}}_{\mathrm{sp}}+\overrightarrow{\mathrm{I}}_{\mathrm{s} 2}$

Where $\vec{I}_{s, \text { in }}\left(\vec{I}_{s, \text { out }}\right)$ is spin current entering $N_{1}$ (exiting $N_{2}$ ). $\vec{I}_{s p}$ is spin current flowing in series branch. $\vec{I}_{\mathrm{s} 1}$ and $\overrightarrow{\mathrm{I}}_{\mathrm{s} 2}$ are dissipated spin currents which are flowed in spin-flip branches. According to spin voltage vector of nodes and spin conduction matrix of $\Pi$ shaped series and spin-flip branches [3]-[7], spin current components of series branch can be represented as

$\mathrm{I}_{\text {sp.x }}=\mathrm{I}_{\mathrm{sp}, \mathrm{y}}=\mathrm{I}_{\mathrm{sp}, \mathrm{z}}=\frac{\mathrm{A}}{\rho \lambda} \operatorname{csch} \frac{\mathrm{L}_{c}}{\lambda_{s}}\left(\mathrm{e}^{\left(-\mathrm{L}_{c} / 2 \lambda_{s}\right)}-\mathrm{e}^{\left(\mathrm{L}_{c} / 2 \lambda_{s}\right)}\right)$

Where $\mathrm{e}^{\left(-\mathrm{L}_{c} / 2 \lambda_{s}\right)}-\mathrm{e}^{\left(\mathrm{L}_{c} / 2 \lambda_{s}\right)}$ is spin voltage difference vector's component Therefore spin current vector of series branch can be represented as

$\overrightarrow{\mathrm{I}}_{\mathrm{s}, \text { out }}=\frac{A}{\rho \lambda}\left[\mathrm{e}^{\left(-\frac{\mathrm{L}}{2 \lambda}\right)}\left(\operatorname{csch} \frac{\mathrm{L}}{\lambda}+\tanh \frac{\mathrm{L}}{2 \lambda}\right)-\mathrm{e}^{\left(\frac{\mathrm{L}}{2 \lambda}\right)} \operatorname{csch} \frac{\mathrm{L}}{\lambda}\right][\hat{\mathrm{x}}+\hat{\mathrm{y}}+\hat{\mathrm{z}}]$

(8)

Also, spin currents components of first and second spin-flip branches are

$\mathrm{I}_{\mathrm{s} 1 . \mathrm{x}}=\mathrm{I}_{\mathrm{s} 1, \mathrm{y}}=\mathrm{I}_{\mathrm{s} 1, \mathrm{z}}=\frac{\mathrm{A}_{c h}}{\rho_{c h} \lambda_{s}} \mathrm{e}^{\left(-\mathrm{L}_{c} / 2 \lambda_{s}\right)} \tanh \frac{\mathrm{L}_{c}}{2 \lambda_{s}}$

$\mathrm{I}_{\mathrm{s} 2 . \mathrm{x}}=\mathrm{I}_{\mathrm{s} 2, \mathrm{y}}=\mathrm{I}_{\mathrm{s} 2, \mathrm{z}}=\frac{\mathrm{A}_{c h}}{\rho_{c h} \lambda_{s}} \mathrm{e}^{\left(-\mathrm{L}_{c} / 2 \lambda_{s}\right)} \tanh \frac{\mathrm{L}_{c}}{2 \lambda_{s}}$

Where $\mathrm{e}^{\left(-\mathrm{L}_{c} / 2 \lambda_{s}\right)}$ and $\mathrm{e}^{\left(-\mathrm{L}_{c} / 2 \lambda_{s}\right)}$ are spin voltage difference components. Therefore spin current of first and second spin-flip branch can be represented as

$\mathrm{I}_{\mathrm{s} 1}=\frac{\sqrt{3} \mathrm{~A}_{c h}}{\rho_{c h} \lambda_{s}} \mathrm{e}^{\left(\mathrm{L}_{c} / 2 \lambda_{s}\right)} \tanh \frac{\mathrm{L}_{c}}{2 \lambda_{s}}$

$\mathrm{I}_{\mathrm{s} 2}=\frac{\sqrt{3} \mathrm{~A}_{c h}}{\rho_{c h} \lambda_{s}} \mathrm{e}^{\left(\mathrm{L}_{c} / 2 \lambda_{s}\right)} \tanh \frac{\mathrm{L}_{c}}{2 \lambda_{s}}$

By conversion a $\Pi$-shaped spin circuit to those of T-shaped, we intend to compute spin current vector in all branches of it (Fig. 2).

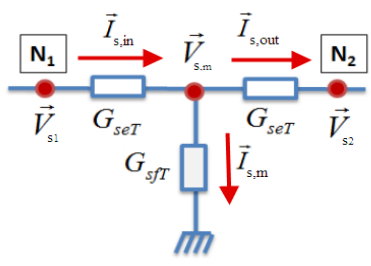

Fig. 2 T-shaped spin circuit with two nano-magnetic nodes and a nonmagnetic channel.

In T-shaped spin circuit, we have middle node which goes to virtual ground. According to Kirchhoff's current, spin current vectors entering middle node and the total dissipated spin current vector at this node as below

$\overrightarrow{\mathrm{I}}_{\mathrm{s}, \text { out }}=\overrightarrow{\mathrm{I}}_{\mathrm{s}, \mathrm{in}}+\overrightarrow{\mathrm{I}}_{\mathrm{s}, \mathrm{m}}$

Where $\vec{I}_{s, m}$ is total dissipated spin current from middle node.

Mathematical forms of $\overrightarrow{\mathrm{I}}_{\mathrm{s} \text {,out }}$ and $\overrightarrow{\mathrm{I}}_{\mathrm{s} \text {,in }}$ are

$\mathrm{I}_{\text {sx }, \text { in }}=\mathrm{I}_{\text {sy,in }}=\mathrm{I}_{\text {sz,in }}=\frac{\mathrm{A}_{c h}}{\rho_{c h} \lambda_{s}}\left[\mathrm{e}^{\left(-\frac{\mathrm{L}}{2 \lambda}\right)} \operatorname{csch} \frac{\mathrm{L}}{\lambda}-\mathrm{e}^{\left(\frac{\mathrm{L}}{2 \lambda}\right)}\left(\operatorname{csch} \frac{\mathrm{L}}{\lambda}+\right.\right.$

$\left.\left.\tanh \frac{\mathrm{L}}{2 \lambda}\right)\right]$

$I_{s x, \text { out }}=I_{\text {sy,out }}=I_{s z, \text { out }}=\frac{A}{\rho \lambda}\left[e^{\left(-\frac{L}{2 \lambda}\right)}\left(\operatorname{csch} \frac{L}{\lambda}+\tanh \frac{L}{2 \lambda}\right)-\right.$

$\left.\mathrm{e}^{\left(\frac{\mathrm{L}}{2 \lambda}\right)} \operatorname{csch} \frac{\mathrm{L}}{\lambda}\right]$

Therefore spin current of these branches can be represented as

$\mathrm{I}_{\mathrm{s}, \text { in }}=\frac{\sqrt{3} \mathrm{~A}_{c h}}{\rho_{c h} \lambda_{s}}\left[\mathrm{e}^{\left(-\mathrm{L}_{c} / 2 \lambda_{s}\right)} \operatorname{csch} \frac{\mathrm{L}_{c}}{\lambda_{s}}-\mathrm{e}^{\left(\frac{\mathrm{L}_{c}}{2 \lambda_{s}}\right)}\left(\operatorname{csch} \frac{\mathrm{L}_{c}}{\lambda_{s}}+\tanh \frac{\mathrm{L}_{c}}{\lambda_{s}}\right)\right]$

$\mathrm{I}_{\mathrm{s}, \text { out }}=\frac{\sqrt{3} \mathrm{~A}_{c h}}{\rho_{c h} \lambda_{s}}\left[\mathrm{e}^{\left(-\mathrm{L}_{c} / 2 \lambda_{s}\right)}\left(\operatorname{csch} \frac{\mathrm{L}_{c}}{\lambda_{s}}+\tanh \frac{\mathrm{L}_{c}}{\lambda_{s}}\right)-\mathrm{e}^{\left(\frac{\mathrm{L}_{c}}{2 \lambda_{s}}\right)} \operatorname{csch} \frac{\mathrm{L}_{c}}{\lambda_{s}}\right]$

Also, spin current components of spin-flip branch is

$\mathrm{I}_{\mathrm{sm}, \mathrm{x}}=\mathrm{I}_{\mathrm{sm}, \mathrm{y}}=\mathrm{I}_{\mathrm{sm}, \mathrm{z}}=\frac{\mathrm{A}_{c h}}{\rho_{c h} \lambda_{s}}\left(\mathrm{e}^{\left(\frac{\mathrm{L}_{c}}{2 \lambda_{s}}\right)}+\mathrm{e}^{\left(-\frac{\mathrm{L}_{c}}{2 \lambda_{s}}\right)}\right) \tanh \frac{\mathrm{L}_{c}}{2 \lambda_{s}}$

Thus spin current can be represented as

$\mathrm{I}_{\mathrm{s}, \mathrm{m}}=\frac{\sqrt{3} \mathrm{~A}_{c h}}{\rho_{c h} \lambda_{s}}\left(\mathrm{e}^{\left(\frac{\mathrm{L}_{c}}{2 \lambda_{s}}\right)}+\mathrm{e}^{\left(-\frac{\mathrm{L}_{c}}{2 \lambda_{s}}\right)}\right) \tanh \frac{\mathrm{L}_{c}}{2 \lambda_{s}}$

\section{Effects of nano-channel length and cross section area variations on spin current}

By using experimental data for copper as nano-channel and permalloy as nano-magnetic nodes [4], spin current has been obtained by taking into account simultaneously effect cross section area and length and results are shown. Fig. 3 shows spin current versus simultaneous variations of cross section area and length nano-channel for series branch of $\Pi$-shaped spin circuit.

According to Fig. 3, length variations have modest effect on spin current reduction while increase of cross section area lead to spin current grow up. Fig. 4 and 5 show dissipated spin current versus cross section area and length variation for spin-flip branch of Пshaped spin circuit. 


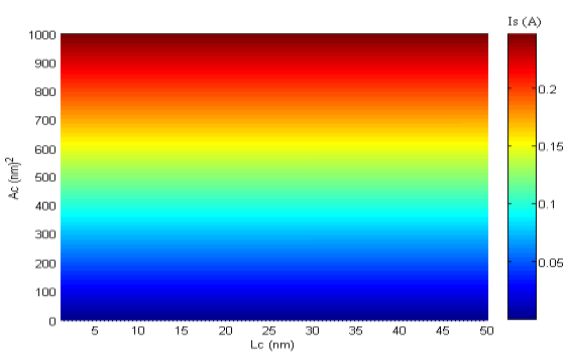

Fig. 3 Spin current versus simultaneous variations of cross section area and length of nano-channel for series branch of П-shaped spin circuit.

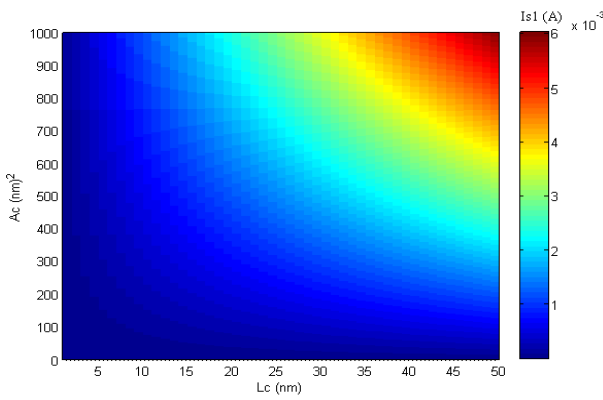

Fig.4Dissipated spin current versus simultaneous variations of cross section area and length of nano-channel for the first spin-flip branch of $\Pi$ shaped spin circuit .

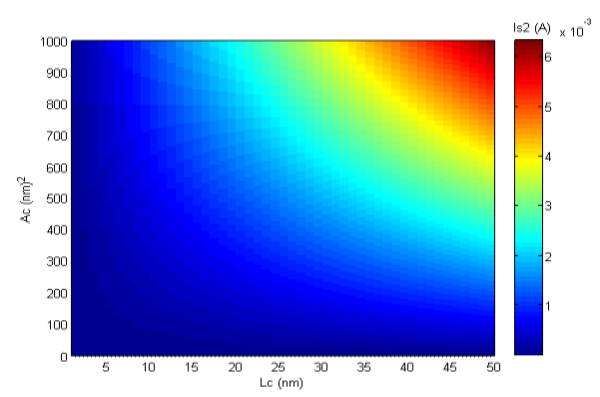

Fig.5 Dissipated spin current versus simultaneous variations of cross section area and length of nano-channel for the second spin-flip branch of П-shaped spin circuit.

According to Figs. 4 and 5, dissipated spin current decrease by cross section area and length reduction. Also, dissipated spin current of first spin-flip branch is larger than those of second because of spin diffusion length. Dissipated spin current decreases by length reduction per specific cross section area. Fig. 6 shows spin current versus nano-channel cross section area and length variations for the first series branch of $\mathrm{T}$-shaped spin circuit.

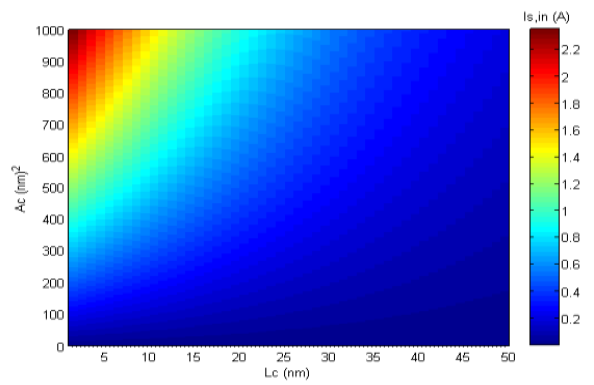

Fig. 6 Spin current versus simultaneous length and cross section area variations related to the first series branch of T-shaped spin circuit.

According to Fig. 6, spin current increases by simultaneously length reduction and cross section area grow up. Spin current increase by length grow up per specific cross section area. Fig. 7 shows spin current versus nano-channel cross section area and length variations for the second series branch of T-shaped spin circuit. Fig. 8 shows dissipated spin current versus the same variations for the spin-flip branch of T-shaped spin circuit.

According to Fig. 7, spin current increase by rise of cross section area and length nano-channel for the series branch of T-shaped spin circuit. Based on Fig. 8, dissipated spin current reduction occurs when we decrease cross section area and length.

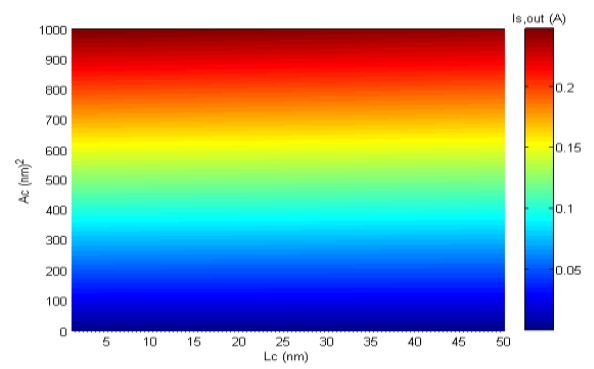

Fig. 7 Spin current versus simultaneous length and cross section area variations related to the second series branch of T-shaped spin circuit.

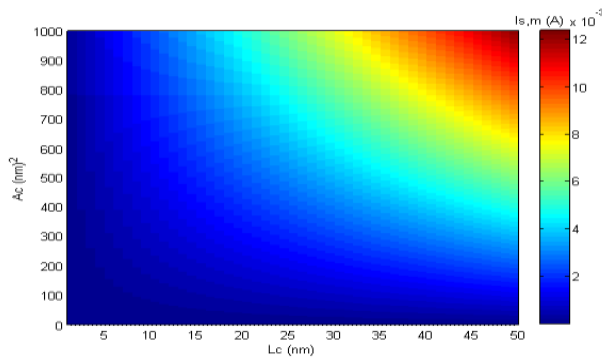

Fig. 8 Dissipated spin current versus simultaneous length and cross section area variations related to the spin-flip branch of T-shaped spin circuit.

Spin current grows up by length reduction for specific cross section area while dissipated spin current is opposed. Tables I and II is illustrated obtained values for spin currents of spin circuit's branches.

Table 1: Obtained values for spin currents related to two branches of $\Pi$ shaped spin circuit.

\begin{tabular}{|c|c|l|l|c|}
\hline \multicolumn{2}{|c|}{ Variants } & \multicolumn{2}{c|}{ spin-flip branch } & Series branch \\
\hline $\mathrm{L}(\mathrm{nm})$ & $\mathrm{A}(\mathrm{nm})^{2}$ & $\mathrm{I}_{\mathrm{s} 1}$ & $\mathrm{I}_{\mathrm{s} 2}$ & $\mathrm{I}_{\mathrm{sp}}$ \\
\hline 20 & 100 & 0.0002 & 0.0002 & 0.03 \\
\hline 30 & 400 & 0.0014 & 0.0015 & 0.1 \\
\hline 50 & 500 & 0.0030 & 0.0031 & 0.13 \\
\hline
\end{tabular}

Table 2: Obtained values for spin currents related to two branches of $\mathrm{T}$ shaped spin circuit.

\begin{tabular}{|c|c|l|l|l|}
\hline \multicolumn{2}{|c|}{ Variants } & \multicolumn{2}{c|}{ Series branch } & spin-flip branch \\
\hline $\mathrm{L}(\mathrm{nm})$ & $\mathrm{A}(\mathrm{nm})^{2}$ & $\mathrm{I}_{\mathrm{s}, \text { in }}$ & $\mathrm{I}_{\mathrm{s}, \text { out }}$ & $\mathrm{I}_{\mathrm{s}, \mathrm{Md}}$ \\
\hline 20 & 100 & 0.02 & 0.26 & 0.004 \\
\hline 30 & 400 & 0.09 & 1.64 & 0.002 \\
\hline 50 & 500 & 0.12 & 2.30 & 0.006 \\
\hline
\end{tabular}

\section{Conclusion}

In this paper, we saw that spin current in series branch increases when we have nano-channel length reduction and nano channel cross section area grow up. As spin current in spin-flip branch shows spin polarization dissipation, we prefer to lower it. We can 
reduce spin current of this branch simultaneously decrease of nano-channel length and cross section area. We choose copper metal choice as nano-channel because of longer spin diffusion length, less spin current dissipation and equality of its lattice constant with those of permalloy.

\section{References}

[1] I. Žutić, J. Fabian, and S. Das Sarma; "Spintronics: Fundamentals and applications"; Rev. Mod. Phys. 76, 323 (2004).

[2] A. Brataas, Y.V. Nazarov, G.E.W. Bauer; "Finite element theory of transport in ferromagnet-normal metal systems"; Phys. Rev. Lett. 84, 24812484 (2000).

[3] S. Srinivasan, A. Sarkar, B. Behin-Aein, and S. Datta; "All Spin Logic Device with Inbuilt Nonreciprocity
Magnetics"; IEEE Transactions on. 47, 4026 (2011).

[4] B. Behin-Aein, A. Sarkar, S. Srinivasan and S. Datta; "Switching energy-delay of all spin logic devices"; Appl. Phys. Lett. 98, 123510 (2011).

[5] Albrecht, J. D., and D. L. Smith, 2002, "Electron spin injection at a Schottky contact," Phys. Rev. B 66, 113303.

[6] Albrecht, J. D., and D. L. Smith, 2003, "Spinpolarized electron transport at ferromagnet/semiconductor Schottky contacts," Phys. Rev. B 68, 035340.

[7] Vlutters, R., O. M. J. van 't Erve, R. Jansen, S. D. Kim,and J. C. Lodder, 2001, "Modeling of spin-dependent hot-electron transport in the spin-valve transistor," Phys. Rev.B 65, 024416. 